\title{
Исследование с помощью молекулярной динамики образования димеров на поверхности (001) GaAs при низких температурах
}

\author{
(C) Н.Д. Прасолов, А.А. Гуткин, П.Н. Брунков \\ Физико-технический институт им. А.Ф. Иоффре Российской академии наук, \\ 194021 Санкт-Петербург, Россия \\ E-mail: nikpras@mail.ioffe.ru
}

Поступила в Редакцию 31 августа 2020 г.

В окончательной редакции 5 октября 2020 г.

Принята к публикации 9 октября 2020 г.

\begin{abstract}
Методом молекулярной динамики с использованием аналитического потенциала, учитывающего $\sigma$ и $\pi$-связи между атомами, выполнено моделирование образования димеров при низкотемпературной реконструкции (001) поверхности GaAs, оканчивающейся атомами Ga или As.

Определены величины уменьшения потенциальной энергии атомов при образовании поверхностного изолированного димера и обнаружено, что потенциальная энергия атома в Аs-димере на несколько десятых эB ниже, чем в Ga-димере. В области температур 25-40 K исследована кинетика начальных этапов образования $\mathrm{Ga}$-димеров и получено, что характеристическая энергия термической активации образования одиночных изолированных Ga-димеров составляет $\sim 29$ мэВ и ниже аналогичной величины для Аs-димеров ( 38 мэВ). В диапазоне температур $28-37 \mathrm{~K}$ оценены постоянные времени, характеризующие среднюю скорость преобразования одиночного димера в цепочку из двух димеров. Для двойных Ga- и As-димеров величины, обратные этим временам, оказались лежащими соответственно в диапазонах $10^{11}-10^{12}$ и $10^{9}-10^{10} \mathrm{c}^{-1}$, тогда как подобные параметры для образования одиночных димеров лежат в диапазоне $4 \cdot 10^{6}-10^{8}$ и $1.4 \cdot 10^{6}-7.4 \cdot 10^{7} \mathrm{c}^{-1}$.
\end{abstract}

Ключевые слова: наноиндентация, реконструкция поверхности, димеры атомов As, димеры атомов Ga, молекулярная динамика.

DOI: 10.21883/FTP.2021.02.50498.9516

\section{1. Введение}

Исследованию свойств поверхности полупроводниковых кристаллов посвящено большое количество экспериментальных и теоретических работ [1,2]. Одним из направлений этих исследований является изучение реконструкции поверхности кристаллов методом молекулярной динамики (МД), в котором применяются различные потенциалы, описывающие взаимодействие атомов в твердом теле. Такой аналитический потенциал, использующий квантово-механические вычисления электронной структуры и учитывающий $\sigma$ - и $\pi$-связи между атомами (bond-order potential - BOP [3]), для кристаллов GaAs был предложен и параметризирован в работе [4]. Вычисленные с его помощью параметры, характеризующие ряд объемных свойств кристаллов GaAs и дефектов в них, находятся в хорошем или удовлетворительном согласии с данными экспериментов и результатами расчетов с помощью теории функционала плотности. Этот потенциал может быть применен и для исследования низкотемпературной реконструкции поверхности GaAs. B ряде экспериментов [2,5] было показано, что реконструкция поверхности (001) GaAs в различных условиях приводит вследствие компенсации оборванных связей к образованию большого количества поверхностных димеров, представляющих собой пары атомов одного и того же типа с уменьшенным межатомным расстоянием.
Эти димеры могут группироваться, образуя различные структуры на поверхности. Начальным этапом реконструкции поэтому является образование поверхностных димеров. Однако кинетика этого процесса практически не исследовалась.

В работе [6] методом МД с применением ВОР были определены начальные скорости и энергия активации низкотемпературного формирования димеров на атомногладкой поверхности (001) GaAs, оканчивающейся атомами As. Представляет интерес изменение этих и других параметров в случае, когда поверхность (001) оканчивается атомами катиона, т. е. Ga. Выяснение этого вопроса является задачей настоящего исследования. С этой целью, кроме определения скоростей и энергии активации образования димеров $\mathrm{Ga}$, для обоих типов поверхности (001) вычислены изменения потенциальной энергии атомов, образующих одиночные изолированный димеры и оценены скорости образования комплексов, состоящих из двух расположенных рядом димеров, т.е. начальные скорости формирования димерных цепочек, вытянутых вдоль направления $\langle 110\rangle$.

\section{2. Методика эксперимента и анализ данных}

Как и в работе [6], при моделировании методом МД мы использовали ВОР, реализованный X.W. Zhow в среде LAMMPS (Large-scale Atomic/Molecular Massively 

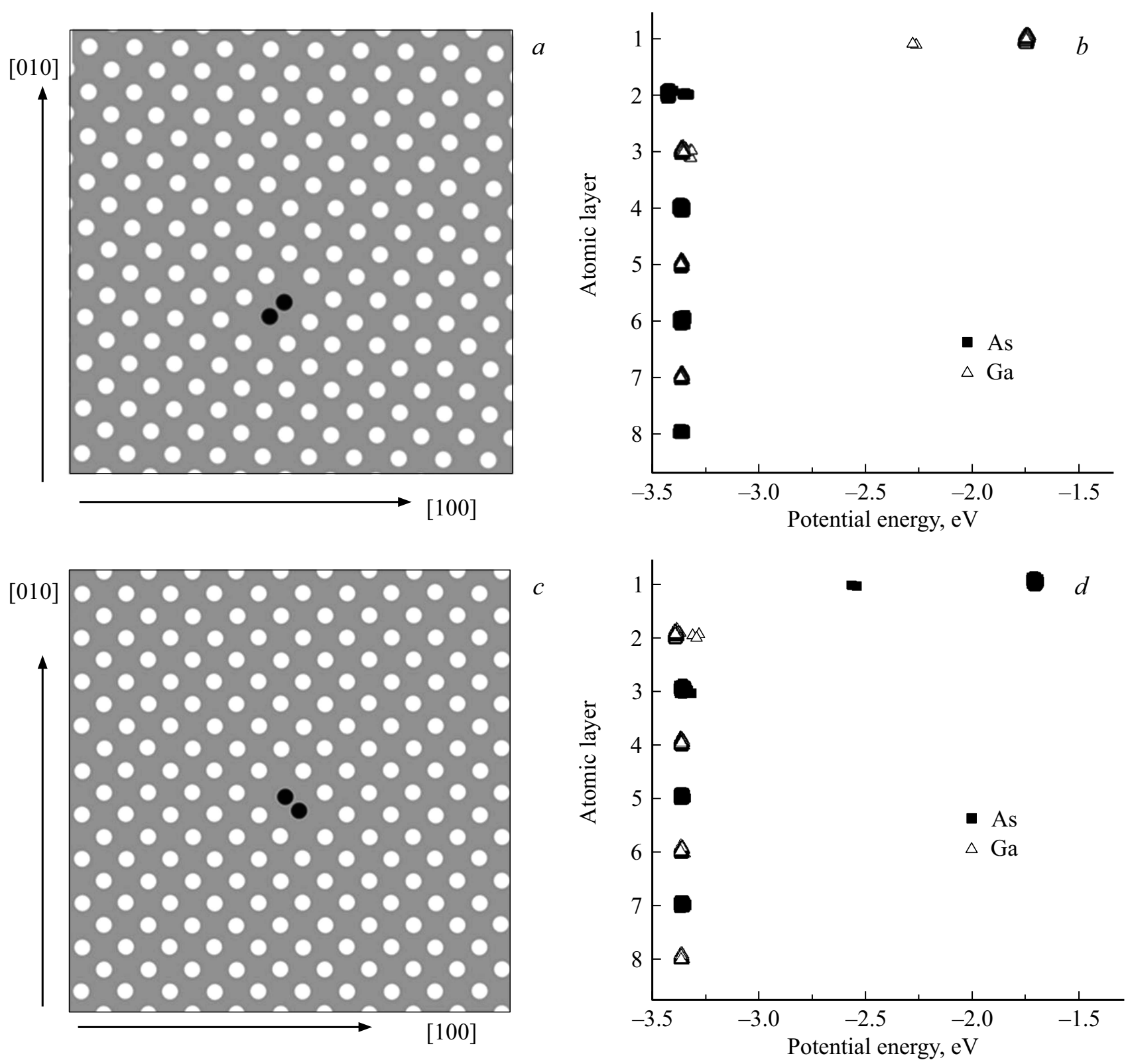

Рис. 1. Расположение одиночных димеров на поверхности и потенциальные энергии атомов в приповерхностных слоях для поверхности GaAs $(001)$, заканчивающихся атомами $\mathrm{Ga}(a, b)$ и As $(c, d) . T=28 \mathrm{~K}$. Черными точками на рис. $a$ и $c$ показаны атомы, образующие одиночный димер.

Parallel Simulator) [7]. Форма, граничные условия и размеры для образца, выбранного при расчетах, также соответствовали работе [6] (высота, ширина и длина соответственно около 12,56 и 56 нм, полное число атомов на поверхности $\left.(001) N_{0}=20000\right)$. В начальный момент времени расположение атомов на поверхности (001) образца полагалось совпадающим с положением атомов в объеме.

Как показали вычисления, в начальной стадии реконструкции поверхности, при образовании изолированного одиночного димера Ga потенциальная энергия каждого поверхностного атома, входящего в димер, становится равной $\sim-2.28$ эВ, т.е. уменьшается на $\sim 0.53$ эВ по сравнению с энергией атома, не образующего димер $(\sim-1.75$ эВ) (рис. $1, b)$. При этом потенциальная энергия связанных с димером атомов в следующем за поверхностью монослое возрастает на величину $\sim 0.1$ эВ. Подобная картина наблюдается и для поверхности (001), оканчивающейся атомами As. Величина потенциальной энергии на каждый атом As, находящийся в одиночном димере, составляет $\sim-2.6$ эВ, а уменьшение этой величины по сравнению с энергией атома, не образовавшего димер $(\sim-1.71$ эВ), составляет $\sim 0.9$ эВ (рис. $1, d)$.

На рис. 2 для $\mathrm{Ga}$ поверхности (001) и низких температур $T$ представлены зависимости относительного числа димеров $N_{\text {rel }}$ от времени $t$, отсчитываемого от момента 
образования свободной поверхности (условного момента скалывания образца):

$$
N_{\text {rel }}(T, t)=\frac{2}{N_{0}} \sum_{i=1} i N_{i}(T, t),
$$

где $N_{i}(T, t)$ димеров каждая. Даже при $N_{\text {rel }} \leq 0.01$ эти зависимости сверхлинейны. Подобная сверхлинейность наблюдалась и для As-поверхности и связывалась с тем, что скорость образования дополнительных димеров у концов цепочек заметно превышает скорость возникновения изолированных димеров [6]. Последнее вызвано изменением потенциала вблизи димера из-за изменения положения поверхностных атомов. При $N_{\text {rel }} \ll 1$ такое изменение существенно только вблизи димера и не существенно на большей части поверхности образца. Тогда начальная скорость образования изолированных димеров может быть определена на основании следующих соображений. Если $N_{\text {rel }}(T, t)$ мало и за промежуток времени от 0 до $t$ не происходит слияния 2 или нескольких цепочек, то полное число изолированных димеров, образовавшихся за это время, $N_{\operatorname{dim} 1}(T, t)$ равно сумме всех цепочек, поскольку каждая новая цепочка возникла из изолированного димера:

$$
N_{\operatorname{dim} 1}(T, t)=\sum_{i=1} N_{i}(T, t) .
$$

С другой стороны, в тех же условиях

$$
N_{\operatorname{dim} 1}(T, t) \approx \frac{N_{0}}{2} \frac{t}{\tau_{1}}
$$

где $\tau_{1}$ - постоянная времени, характеризующая среднюю начальную скорость образования изолированных димеров.

Линейная аппроксимация (3) результатов расчетов величин $N_{i}(T, t)$ для димеров Ga показана на рис. 3 , а полученные при этом значения $\tau_{1}^{-1}$ сопоставляются с

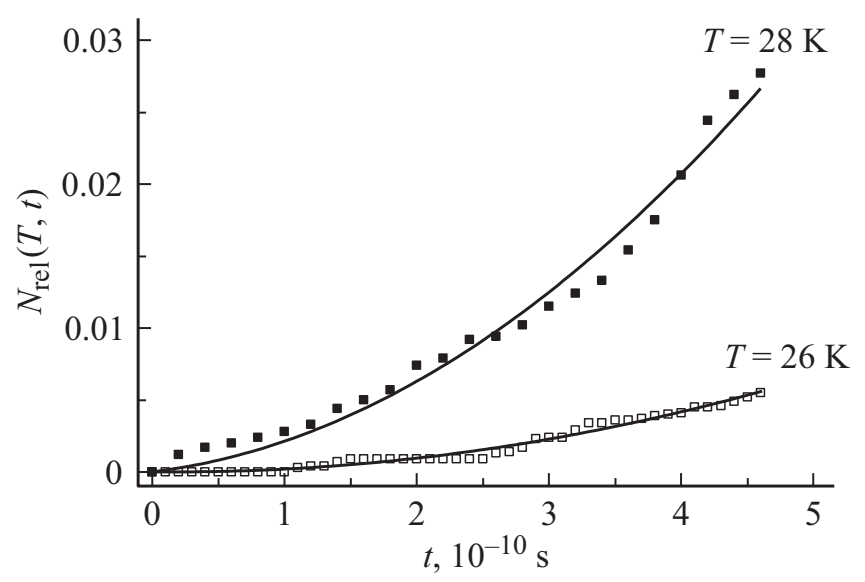

Рис. 2. Зависимость относительного числа поверхностных димеров $\mathrm{Ga} N_{\text {rel }}(T, t)$ от времени $t$, отсчитываемого от момента образования свободной поверхности (скалывания образца).

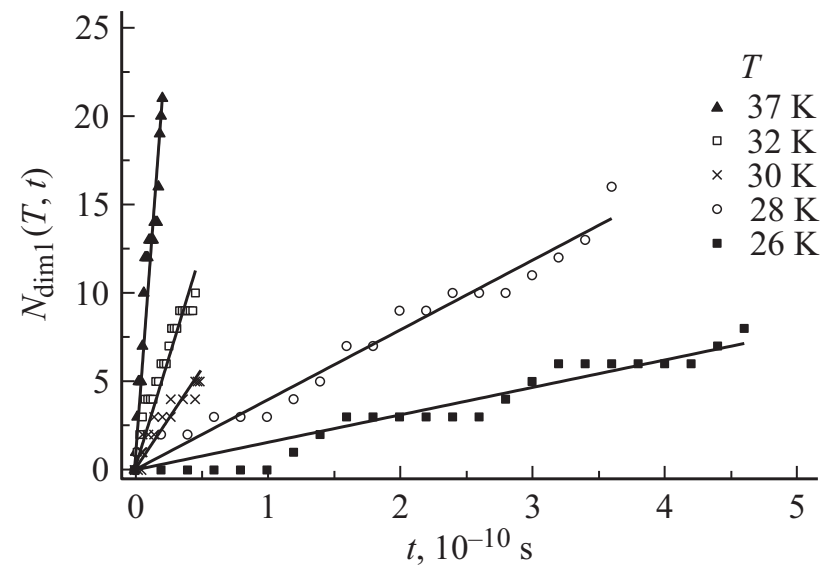

Рис. 3. Зависимости $N_{\operatorname{dim} 1}(T, t)$ от времени $t$ при разных температурах для поверхности GaAs (001), оканчивающейся атомами Ga.

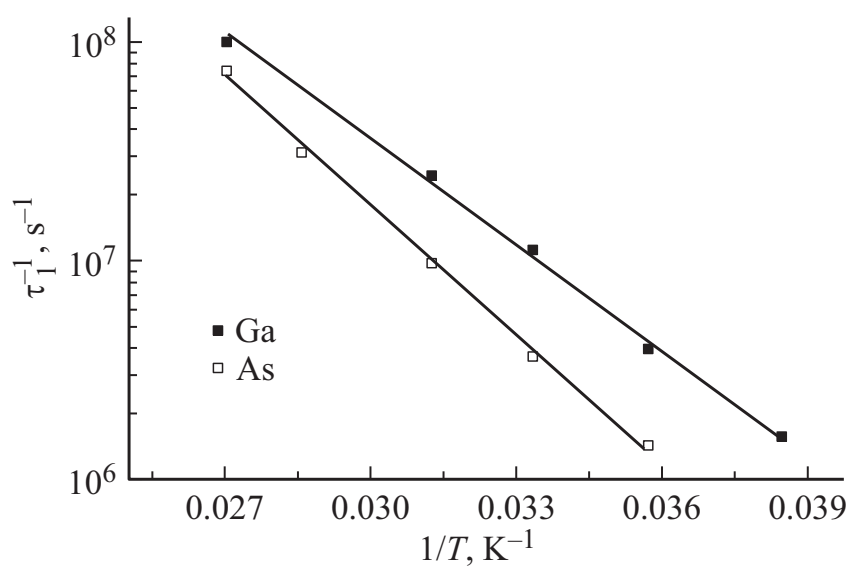

Рис. 4. Зависимости от температуры постоянных времени $\tau_{1}$, характеризующих среднюю начальную скорость образования изолированных поверхностных димеров As [6] и $\mathrm{Ga}$.

данными для димеров As [6] на рис. 4. Энергия активации образования изолированных димеров $\mathrm{Ga}$ составляет $\sim 29$ мэВ, тогда как соответствующая величина для As равна $\sim 38$ мэВ.

Аналогичные соображения могут быть использованы и для оценки постоянной времени $\tau_{2}$, характеризующей начальный этап образования цепочек из двух димеров. Этот процесс осуществляется путем формирования одиночного димера из атомов, находящихся в непосредственном соседстве с атомами уже существующего изолированного димера. Число таких цепочек, создающихся в промежуток времени от 0 до $t, N_{\operatorname{dim} 2}(T, t)$ равно:

$$
N_{\operatorname{dim} 2}(T, t)=\sum_{i=2} N_{i}(T, t),
$$

а среднее начальное изменение этой величины со временем описывается соотношением:

$$
\frac{d N_{\operatorname{dim} 2}(T, t)}{d t}=\frac{2 N_{1}(T, t)}{\tau_{2}},
$$


T.e.

$$
N_{\operatorname{dim} 2}(T, t)=\frac{2 \int_{0}^{t} N_{1}(T, t) d t}{\tau_{2}} .
$$

Коэффициент 2 перед интегралом означает, что при образовании двухдимерной цепочки дополнительный димер может возникать как с одной, так и с другой стороны изолированного димера.

Анализ результатов вычислений $N_{i}(T, t)$ с помощью выражений (4) и (5) в диапазоне изменения $\sum_{i=2} N_{i}(T, t)$ от 0 до $\sim 20$ показывает, что при температурах $28-37 \mathrm{~K}$ значения $\tau_{2}^{-1}$ более, чем на 2 порядка превышают значения $\tau_{1}^{-1}$ и лежат в интервале $10^{11}-7 \cdot 10^{11}$ и $3 \cdot 10^{9}-10^{10} \mathrm{c}^{-1}$ соответственно для димеров Ga и As.

\section{3. Заключение}

Таким образом, моделирование низкотемпературного образования димеров на свободной поверхности (001) с использованием аналитического потенциала [4,7] показывает, что величина уменьшения потенциальной энергии поверхностного атома при образовании одиночного изолированного димера As больше, чем при образовании димера Ga. B расчете на один такой атом эти величины соответственно равны $\sim 0.9$ и $\sim 0.53$ эВ. При этом постоянная времени, характеризующая начальную среднюю скорость образования изолированных димеров в диапазоне температур 26-37 K, для атомов Ga заметно меньше, чем для атомов As. Энергия термической активации этого образования составляет $\sim 29$ мэВ, а величина, обратная характеристическому времени, при увеличении температуры от 28 до $37 \mathrm{~K}$ растет в интервале $4 \cdot 10^{6}-10^{8} \mathrm{c}^{-1}$, тогда как эти параметры для димеров As имеют величины $\sim 38$ мэВ и $1.4 \cdot 10^{6}-7.4 \cdot 10^{7} \mathrm{c}^{-1}$. В диапазоне температур $28-37 \mathrm{~K}$ постоянная времени, характеризующая начальную среднюю скорость преобразования изолированного димера в цепочку из двух димеров, на несколько порядков ниже соответствующей величины для образования изолированных димеров. Величины, обратные этому времени для цепочек из димеров $\mathrm{Ga}$ и As, находятся соответственно в диапазонах $10^{11}-10^{12}$ и $10^{9}-10^{10} \mathrm{c}^{-1}$.

\section{Конфликт интересов}

Авторы заявляют, что у них нет конфликта интересов.

\section{Список литературы}

[1] V.P. LaBella, H. Yang, D.W. Bullock, P.M. Thibado, P. Kratzer, M. Shcheffler. Phys. Rev. Lett., 83, 2989 (1999).

[2] V.P. LaBella, M.R. Krause, Z. Ding, P.M. Thibado. Surf. Sci. Rep., 60, 1 (2005).

[3] D.G. Pettifor, I.I. Oleinik. Phys. Rev. B, 59, 8487 (1999).
[4] D.A. Murdick, X.W. Zhow, Y.N.G. Wadley, D. Nguyen-Manh, R. Drautz, D.G. Pettifor. Phys. Rev. B, 73, 045206 (2006).

[5] A. Ohtake. Surf. Sci. Rep., 63, 295 (2008).

[6] N.D. Prasolov, A.A. Gutkin, P.N. Brunkov. J. Phys. Conf. Ser., 1410, 012225 (2019).

[7] http://lammps.sandia.gov/doc/pair_bop.html

Редактор Г.А. Оганесян

\section{Molecular dynamics study of dimer formation on GaAs (001) surface at low temperatures}

\section{N.D. Prasolov, A.A. Gutkin, P.N. Brunkov}

loffe institute, 194021 St. Petersburg, Russia

Abstract The simulation of dimers formation during the lowtemperature reconstruction of GaAs (001) surface terminated with Ga or As atoms was performed by the molecular dynamics method using the analytical Bond-Order Potential based on quantum mechanical theory incorporating both $\sigma$ - and $\pi$-bonds between atoms.

A decrease in values of potential energy of the atoms during formation of isolated surface dimer have been determined. It has been found that potential energy of an atom in As-dimer is several tenths of an $\mathrm{eV}$ lower than in Ga-dimer. Kinetics of the initial stages of Ga-dimers formation in the temperature range of $25-40 \mathrm{~K}$ was studied. It was found that the characteristic thermal activation energy of single isolated Ga-dimers formation is $\sim 29 \mathrm{meV}$, which is lower than the same value for As-dimers $(\sim 38 \mathrm{meV})$. Time constants characterizing the average rate of transformation of one dimer into a chain of two dimers at temperature range of $28-37 \mathrm{~K}$ were estimated. Inverse values of these parameters for paired Ga- and As-dimers are in the ranges of $10^{11}-10^{12}$ and $10^{9}-10^{10} \mathrm{~s}^{-1}$, respectively, while corresponding parameters for the formation of single dimers are in the ranges of $4 \cdot 10^{6}-10^{8}$ and $1.4 \cdot 10^{6}-7.4 \cdot 10^{7} \mathrm{~s}^{-1}$. 